\title{
The Tibetan Dance "Mother" Brought To The Dance Of The Enlightenment
}

\author{
Yutong Shan \\ College of music and dance, Qujing Normal University, Qujing, Yunnan, 655011 \\ qjsfxysyt@126.com
}

Keywords: Tibetan Dance; "Mother"; Dance Creation; Art Appreciation; Inspiration

\begin{abstract}
Abtract. The Tibetan Dance "mother", by shaping the image of mother's mother is the kind of strong and have experienced years of wind and frost. The great mother singing, for the mother of the artistic image of accurate characterization, through the art of dance language display. As a successful creation of artistic works, the image of the mother, mainly through the dance movements, stage costumes, actors and emotional expression and music background rendering and so on, the aesthetic characteristics of the dance show. This paper analyzes the successful elements of Tibetan Dance "mother", and tap the artistic innovation.
\end{abstract}

\section{Introduction}

Tibetan social history has a long history, thousands of years of accumulated culture is a long time. Especially the ecological elements of Tibetan culture, but also other forms of dance art is not. The success of the Tibetan Dance "mother" is that it not only shows the most universal significance of the Tibetan life history, but also reveals the characteristics of the Tibetan people and the unique aesthetic feelings of the people in the art language. When we watch the Tibetan Dance "mother", from the perspective of the appreciation of art works to enjoy the Tibetan culture, but also from the elements of the dance to feel the Tibetan people's lives revealed by the strong national flavor [1].

\section{Tibetan Dance Art Expression Form}

Tibetan Dance is A Folk Dance. Tibetan Dance "mother" is completed by the famous Chinese director Zhang Jigang creation, the main content of the dance, a Tibetan mother image out of shape. The main performer for Tibetan dancers Dolma, the show to itself with Tibetan characteristics of expression, in the form of dance behave hearty [2]. From the academic point of view of the folk dance in China can be divided into "harmony" and "Zhuo" two types. Tibetan dance in the form of a collective dance, so the Tibetan folk in the form of "harmony" in the form of. Tibetan dance after the long-term development, integration of a variety of cultural elements, some basic dance movements into upper limb movements, the collective formation of transform and in-situ rotation of the dance form is embodied into the Tibetan dance creation, making the Tibetan Dance "harmonious" expressed more diverse and perfect [3].

The Folk and Religious of the Tibetan Dance. Tibetan dance originated from the long-term production of the Tibetan people, so it has the folk; the Tibetan, as the Chinese minority, it has its own religious beliefs, therefore, in the dance creation, it is bound to show religious color [4]. Folk and Tibetan dance is often created in the busy amuse oneself, and therefore more relaxed, cheerful, and was accepted by the public. The religion of the Tibetan dance into a more rich cultural, religious color, which, in a graceful dance posture show. Whether it is a folk dance, or a religious dance, there is a common feature between the two, and from the expression of the stage art, you can from the performer's costume features, performance form to distinguish [5].

The Characteristics of Tibetan Dance. Tibetan is the national life in plateau area, all the year round living in high altitude and low oxygen environment, life activities to from the turnover in the mountain, the iterative process. Long and carrying heavy loads on the mountain, the exercise of the Tibetan people's knee joint. Therefore, on the performance of the Tibetan dance, the work of the knee joint. With the rhythm of the music, the dance is more with the rhythm of the music. So in the 
appreciation of Tibetan dance, feel this seemingly leisurely, but bursting with passion and enjoyable.

Tibetan dance in the form of characteristics, mainly outside the open for the United states. By the Tibetan dance movements analysis, leg lift, leg and kick and cross back and left leg and so on, pay attention to open the form of beauty, but more emphasizes the coordination of the dance movements. For example, with the same hand foot action in order to give people a sense of dislike. However, there is such a creation of dance movements in the Tibetan dance, and it can be maintained in a high degree of coordination. From the expression of the dance, the woman is more elegant and reserved, while the man show is bold beauty. Generally speaking, the women's movement in the Tibetan dance in the past, the vertical arm is the main dance form features, this action language is consistent with the characteristics of the Tibetan women. Man on expression of Tibetan dance movements, based mainly on the aspectual properties of the pitman arm, a breakdown of the hip, but in order to give to open-minded, cheerful feeling [6].

\section{The Aesthetic Characteristics of Tibetan Dance "Mother"}

The Aesthetic Features of Tibetan Dance "Mother" in the Action Form. The dance is a kind of visual art, which is based on the artistic form of the mother's image, and it should be in accordance with the characteristics of Tibetan life. On the other hand, it is more in accordance with the artistic expression of the Tibetan. Mother's dance image building to collapse waist Qiaotun "as the main characteristics, in line with the Tibetan Dance women before tilting movements characteristic [7]. Tibetan Dance "mother" of the leading dancer is Tibetan dancers, in physical characteristics can be showing the Tibetan women's unique morphological features, coupled with its action in the dance "the collapse of waist Qiaotun", mother that bear the burden of life, have to often labor year image out of shape, at the same time, the mother's role is more capable of demonstrating the mother of endoplasmic mother, old, but kind and gentle. Mother's kind of simplicity, but also from the show out. Although this action can not give people a sense of a single, but it is precisely this kind of creation, making the Tibetan Dance "mother" of the creation of more clear. Tibetan Dance "mother" mother image in dance expression mainly adopts the Tibetan dance in the spring. The form of the outer form is the change of the arm and the change of the foot.

The Aesthetic Features of Tibetan Dance "Mother" in the Emotion of the Dance. Although the dance art acts, but the dance is another form of emotional. In the Tibetan Dance "mother", to promote the dance style through the dance always, and the typical mother image like stands where the sculpture as eternal, the burden of life needs a mother bear, the premature mother spine bends down, secretly crooked figure is a kind of stage art image, is deeply engraved into the audience's mind. Static art expression in the Tibetan dance, it seems that the dynamic of the notes suddenly static, the audience's thinking is suspended, but not in the form of a blank, but from the subjective thought, the connotation of the dance to be extended, making the moment to stop the notes

\section{The Aesthetic Features of the Tibetan Dance "Mother" in the Clothing and Music}

Dance art is a language to express the meaning of dance and dance creators, however, based on its expression form is not a single existence, therefore, dance art has a comprehensive feature. "Mother" in Tibetan dance for the mother image positioning in the "ragged jacket" artistic imagination. Visible mother pay too much for a living, but is precisely the living environment, the shape of the mother was able to bear hardships and hardworking as spirit [9]. Music as the voice of the dance, rendering the dance, making the dance very expressive, and strengthen the stage of infection.

Dance is perceptual, the content of the shape is always in the form of abstract implicit expression. In the mother's dance art image design, music runs through the whole work, so that the expression of the idea of the dance is more accurate in place. If the dance is a form of music, then the dance can be use music expression, passionate depiction of characters of the character display. The 
creation of the artistic image of the Tibetan mother, from a certain sense, it is the ideological and emotional shape. The idea into the dance, making the mother's image more vivid.

Three, the Tibetan Dance "'" mother of the success of the creation of the enlightenment

The Success of the Theme of the Dance Works is the Primary Condition. In the creation of dance, in order to produce a good artistic effect, it is often to focus on the theme of the dance for the performance of its positioning, and identify the entry point, the creation of a knowledge structure has become an important factor. Dance works of good and bad, involves the creation of knowledge structure, the creation of the calendar year and the psychological factors, also for the dance theme of geographical environment and customs to has a deep understanding of, and understand, and can only be expressed accurately in the theme of the dance creation will dance. In dance creation, to experience the material, structure and choreography creation steps three.

Tibetan Dance "mother" in the selection has been very clear, the creators should focus on building a dance structure. Dance structure is different from other artistic creation, this is a psychological process, whether in the design of the dance, or dance choreography, which are related to the theme of the dance more profound ideas and sublimation [10]. Dance is sensitive, not only in the performance of the dance, but also reflected in the creation of. The sensitivity of dance creation is determined by the creator's thinking of the expression form of the dance theme, which will be formed by the influence of various factors, and will be incorporated into the dance creation.

Innovative elements in the creation of the dance, which is the important element of the success of the Tibetan Dance "mother". That is to say, in the creation of the dance, it is needed to inject some fresh blood into the subject. Tibetan dance and Tibetan people's life is closely related to the dance itself originated in the folk, so the Tibetan dance and not to give people a sense of, but, to let the "mother" of the theme is more distinctive, outstanding, and the creators of the idea to express, you need to add fresh blood, so that the dance performance based on the audience, but also in thinking and emotional resonance. From the Tibetan Dance "mother" expression of the theme, it is to use a special form of dance art in the form of mother, then, mother's kindness, mother's strong will be expressed in the art form of dance, but to dance performers play, produce a sense of intimacy, so that the theme of the work of the audience, the formation of the awareness of the image of tibetan. Dance to the hearts of the people, to be able to accurately convey the inner thoughts. Therefore, the Tibetan Dance "mother" in the creation of the theme around the theme, in addition to the mother's art image positioning, and integrated into the idea of the creator to express.

The Perfect Interpretation of the Dance Art Requires the Proper Dance Actors to Support. From an aesthetic point of view, dance art is the art of human movement, which is a clever use of the artistic movements and various skills, the content of the dance to express. But if the dancers in acting, just stick to dance moves and skills, but not for the content of the dance to a deep understanding of, can show out the rhythm and rich aesthetic picture, is empty and without meaning. The expression of the dance art is the creation of the stage again, the dancers in the process of performing, is based on the profound understanding of the work and the formation of the ideological expression, through the dance language. Visible, dance performer, but also the creator of stage art.

Tibetan Dance "mother" by the famous Tibetan dancer Zhuoma show. Zhuoma grew up in the Tibetan areas, the Tibetan life has become a profound basis, and formed a dance image of the material basis. As a member of the Tibetan ethnic group, Zhuoma and his mother on stage performance is not the image of the mother, but also to show the living conditions of the mother in the Tibetan life, and Zhuoma's dance performance, in fact, is the real life of the Tibetan mother of an art. Various social experience and experience, have become the important elements of dance performances, makes the image of a Tibetan mother out of the spirit is highly expressed and its connotation by Zhuoma delicate work and shrewdness and eyes can be released. Visible, to the Tibetan Dance "mother" of the artistic imagination to express accurately, appropriate actor, proper deduction is very important, through the quality of the actor's art and life understanding, the spirit of the works expressed, coupled with creators novel creative dance, dance of a successful work on the establishment of in the strong artistic impact based on, has been a large number of audience. 


\section{Conclusion:}

In summary, it is said that dance is a poem, it is said that the dance is the flow of painting. Dance as an action art, which is based on human long-term activities and the accumulation of artistic practice, which integrated into the artistic aesthetic elements. So, the dance is an experience, and abstract. In the creation of the Tibetan Dance "mother", the creator from the point of view of experience for Tibetan mother image to be positioned the abstract art, infiltration of their own ideas, coupled with dance performers, as the Tibetan people, long-term since the formation of aesthetic point of view, customs and religious beliefs and daily modes of production and life, all create a unique artistic style of the Tibetan dance, its unique charm.

\section{References}

[1] Cai Chuan. Basic Characteristics of Tibetan Dance and Its Innovation and Development. Art \& Technology. 2014 (02).182-182.

[2] Fu Xiao. On The Dance Creation Psychological Process. Shandong Youth Political College Journal.2011 (05).143-145.

[3] Liu Hongi. The Science of Tibetan Dance. 2010 (12).120-121.

[4] Koto. Tibetan Original Dance Cultureof. National Art Research.2013 (02) 53-56,66.

[5] Yang. The Basic Characteristics and The Innovation And Development of Tibetan Dance Research. Jilin Province Institute of.2013 (07) 34-35..

[6] Yang Fan, Wang-Long S. The Success of The Tibetan Dance Mother on The Dance Creation of The. Art Technology.2014 (03).190. 